\title{
Spectroscopy of Element 115 Decay Chains
}

\author{
D. Rudolph, ${ }^{1, *}$ U. Forsberg, ${ }^{1}$ P. Golubev, ${ }^{1}$ L. G. Sarmiento, ${ }^{1}$ A. Yakushev, ${ }^{2}$ L.-L. Andersson, ${ }^{3}$ A. Di Nitto, ${ }^{4}$
} Ch. E. Düllmann, ${ }^{2,3,4}$ J. M. Gates, ${ }^{5}$ K. E. Gregorich, ${ }^{5}$ C. J. Gross, ${ }^{6}$ F. P. Heßberger, ${ }^{2,3}$ R.-D. Herzberg, ${ }^{7}$ J. Khuyagbaatar, ${ }^{3}$ J. V. Kratz, ${ }^{4}$ K. Rykaczewski, ${ }^{6}$ M. Schädel, ${ }^{2,8}$ S. Åberg, ${ }^{1}$ D. Ackermann, ${ }^{2}$ M. Block, ${ }^{2}$ H. Brand, ${ }^{2}$ B. G. Carlsson, ${ }^{1}$ D. Cox, ${ }^{7}$ X. Derkx,${ }^{3,4}$ K. Eberhardt, ${ }^{3,4}$ J. Even, ${ }^{3}$ C. Fahlander, ${ }^{1}$ J. Gerl, ${ }^{2}$ E. Jäger, ${ }^{2}$ B. Kindler, ${ }^{2}$ J. Krier, ${ }^{2}$ I. Kojouharov, ${ }^{2}$ N. Kurz, ${ }^{2}$ B. Lommel, ${ }^{2}$ A. Mistry, ${ }^{7}$ C. Mokry, ${ }^{3,4}$ H. Nitsche, ${ }^{5}$ J. P. Omtvedt, ${ }^{9}$ P. Papadakis, I. Ragnarsson, ${ }^{1}$ J. Runke, ${ }^{2}$ H. Schaffner, ${ }^{2}$ B. Schausten, ${ }^{2}$ P. Thörle-Pospiech, ${ }^{3,4}$ T. Torres, ${ }^{2}$ T. Traut, ${ }^{4}$ N. Trautmann, ${ }^{4}$ A. Türler, ${ }^{10}$ A. Ward, ${ }^{7}$ D. E. Ward, ${ }^{1}$ and N. Wiehl ${ }^{3,4}$

${ }^{1}$ Lund University, 22100 Lund, Sweden

${ }^{2}$ GSI Helmholtzzentrum für Schwerionenforschung GmbH, 64291 Darmstadt, Germany

${ }^{3}$ Helmholtz Institute Mainz, 55099 Mainz, Germany

${ }^{4}$ Johannes Gutenberg-Universität Mainz, 55099 Mainz, Germany

${ }^{5}$ Lawrence Berkeley National Laboratory, Berkeley, California 94720, USA

${ }^{6}$ Oak Ridge National Laboratory, Oak Ridge, Tennessee 37831, USA

${ }^{7}$ University of Liverpool, Liverpool L69 7ZE, United Kingdom

${ }^{8}$ Advanced Science Research Center, Japan Atomic Energy Agency, Tokai, Ibaraki 319-1195, Japan

${ }^{9}$ University of Oslo, 0315 Oslo, Norway

${ }^{10}$ Paul Scherrer Institute and University of Bern, 5232 Villigen, Switzerland

(Received 11 June 2013; published 10 September 2013)

\begin{abstract}
A high-resolution $\alpha$, x-ray, and $\gamma$-ray coincidence spectroscopy experiment was conducted at the GSI Helmholtzzentrum für Schwerionenforschung. Thirty correlated $\alpha$-decay chains were detected following the fusion-evaporation reaction ${ }^{48} \mathrm{Ca}+{ }^{243} \mathrm{Am}$. The observations are consistent with previous assignments of similar decay chains to originate from element $Z=115$. For the first time, precise spectroscopy allows the derivation of excitation schemes of isotopes along the decay chains starting with elements $Z>112$. Comprehensive Monte Carlo simulations accompany the data analysis. Nuclear structure models provide a first level interpretation.
\end{abstract}

DOI: 10.1103/PhysRevLett.111.112502

Citius, altius, fortius.-The Olympic motto translated to the nuclear landscape calls for the quest of new isotopes at its outskirts, which is particularly true at its upper end: here, the first observation of a new isotope may imply the specifically rewarding first observation of a new chemical element.

During the past decade, a number of correlated $\alpha$-decay chains, which all terminate by spontaneous fission, have been observed in several experiments using ${ }^{48} \mathrm{Ca}$-induced fusion-evaporation reactions on actinide targets $[1,2]$. The collaborations working at the Flerov Laboratory of Nuclear Reactions (FLNR) in Dubna, Russia, interpret these to originate from the production of isotopes with proton numbers $Z=112-118$ [3].

In recent years, decay chains consistent with even- $Z$ data from FLNR were independently observed [4-10]. In 2011, the discovery of elements $Z=114$ (flerovium) and $Z=116$ (livermorium) was acknowledged [11]. However, assignments of $Z$ and mass numbers $A$ of these superheavy elements rely on indirect techniques, such as measurements of excitation functions, cross bombardments, or $\alpha$-decay systematics, which all depend largely on nuclear mass models. Additional evidence-eventually direct or independentfor $Z$ and $A$ is being requested for claims on elements $Z=113,115,117$, and 118 [11].
PACS numbers: 21.10.-k, 23.20.Lv, 23.60.+e, 27.90.+b

Alpha decay of odd- $A$ or odd-odd nuclei typically proceeds to excited states in the daughter nucleus, because unpaired nucleons tend to remain in the same single-particle orbitals as in the $\alpha$-decay parent [12]. Observation of electromagnetic decays from these excited states can thus elucidate the low-lying nuclear structure of the daughter [13]. Electromagnetic decay into lower-lying states can also proceed by internal conversion, and following the emission of the conversion electron from the atom, $\mathrm{x}$-ray emission is by far the most likely process in the atomic deexcitation. The energy of characteristic $\left(K_{\alpha 1}, K_{\alpha 2}\right.$, and $\left.K_{\beta}\right)$ x rays is one direct measure of $Z$ [14]. These energies are predicted with high accuracy (e.g., Refs. [15-18]) and are consistent with experimental data existing up to $\mathrm{Db}(Z=105)$ [19]. The atomic number of rutherfordium $(Z=104)$ was determined by x-ray spectroscopy in the early 1970s [20].

Here we report on the first such nuclear spectroscopy experiment and $\mathrm{x}$-ray fingerprinting attempt along $\alpha$-decay chains anticipated to start from element $Z=115$.

The Universal Linear Accelerator at the GSI Helmholtzzentrum für Schwerionenforschung, Darmstadt, Germany, provided typically $6 \times 10^{12}{ }^{48} \mathrm{Ca}^{8+}$ ions per sec (time averaged over its pulsed structure, $5 \mathrm{~ms}$ beam on and $15 \mathrm{~ms}$ beam off) at energies of 5.400 and $5.462 \mathrm{MeV} / u$. 
The beam impinged on a rotating target wheel, which comprised four target segments averaged $0.83(1) \mathrm{mg} / \mathrm{cm}^{2}$ ${ }^{243} \mathrm{Am}_{2} \mathrm{O}_{3}$. At Mainz University, the ${ }^{243} \mathrm{Am}$ material from Oak Ridge was electroplated onto 2.20(5) $\mu \mathrm{m}$ thick titanium backing foils. These foils faced the beam, which implies midtarget beam energies of 242.1 and $245.0 \mathrm{MeV}$. Beam integrals of 2.13(12) and 3.89(23) $\times 10^{18}{ }^{48} \mathrm{Ca}$ ions were collected, respectively.

The target wheel was positioned at the entrance of the recoil separator TASCA $[9,21,22]$, filled with He gas at a pressure of $p_{\mathrm{He}}=0.8 \mathrm{mbar}$ [23]. For the major portion of the experiment, TASCA was set to center ions in the focal plane with a magnetic rigidity of $B \rho=2.21 \mathrm{Tm}$. These ions entered the multicoincidence spectroscopy setup TASISpec [24]. Based on earlier in-beam calibrations and simulations, the efficiency for element $Z=115$ fusionevaporation residues was estimated to $30(3) \%[25,26]$.

TASISpec comprises a $32 \times 32$-strip double-sided silicon strip detector (DSSSD), $6 \times 6 \mathrm{~cm}^{2}$ in area and $0.52 \mathrm{~mm}$ thick. A cubelike detector arrangement is formed by four additional $16 \times 16$-strip DSSSDs of the same size and placed upstream. The 96 preamplified signals from the $n$-doped sides of the DSSSDs were processed with standard electronics [24], while 70- $\mu$ s long traces of the preamplified signals of all $p$-doped sides, which are most relevant for high-resolution spectroscopy, were digitized by $60 \mathrm{MHz}, 12$-bit sampling ADCs. Five composite germanium detectors were placed closely behind each of the five DSSSDs. Their signals were handled by $100-\mathrm{MHz}, 16-$ bit sampling ADCs. Baseline and flat-top energies and time were recorded, eventually flagged with pileup recognition.

List mode data were trigger generated by a coincident signal from a $p$-side and $n$-side strip of the implantation detector, the latter limiting the trigger threshold to some $500 \mathrm{keV}$ energy deposited. Trigger rates were typically $100-120$ events per sec. Beam on-off status and irradiated target segment number were recorded. Upon detection of an 8.5-11.0 MeV particle in the implantation DSSSD during beam-off periods, the ${ }^{48} \mathrm{Ca}$ beam was chopped within $<20 \mu \mathrm{s}$ for a period $\Delta t_{1}$. If within $\Delta t_{1}$ another event occurred in the same detector strip at time $t_{2}$, the beam-off period was extended for a period $\Delta t_{2}$ starting at $t=t_{2}$. Pairs of $\left(\Delta t_{1}, \Delta t_{2}\right)=(5,30 \mathrm{~s}),(20,40 \mathrm{~s})$, and $(20,60 \mathrm{~s})$ were used.

During the offline data analysis, emphasis has been put on spectroscopic precision. To name but a few, this includes individually optimized preamplifier decay constants for the moving-window algorithm of the sampled DSSSD pulses, self-consistent pixel-by-pixel determination of DSSSD dead layer thicknesses based on electron and $\alpha$ source data as well as known $\alpha$-decay energies and $Q_{\alpha}$ values of background radiation from transfer reaction products. More details can be found in Ref. [27].

A search for time- and position-correlated recoil- $\alpha-\alpha$ and recoil- $\alpha$-fission sequences was conducted using (i) $12.0<E_{\text {rec }}<18.0 \mathrm{MeV}$, beam on;

(ii) $9.0<E_{\alpha 1}<12.0 \mathrm{MeV}, \Delta t_{\text {rec- } \alpha 1}=5 \mathrm{~s}$, beam off, or $10.0<E_{\alpha 1}<12.0 \mathrm{MeV}, \Delta t_{\text {rec }-\alpha 1}=1 \mathrm{~s}$, beam on;

(iii) $9.0<E_{\alpha 2}<11.0 \mathrm{MeV}, \Delta t_{\alpha 1-\alpha 2}=20 \mathrm{~s}$, beam off, or $9.5<E_{\alpha 2}<11.0 \mathrm{MeV}, \Delta t_{\alpha 1-\alpha 2}=5 \mathrm{~s}$, beam on;

(iv) $E_{\mathrm{SF}}>120 \mathrm{MeV}, \Delta t_{\alpha 1-\mathrm{SF}}=30 \mathrm{~s}$, beam off.

During beam-off and background measurement periods 64 fission events occurred. Most of these were correlated with one of the thirty $\alpha$-decay chains found via the search algorithm, or associated with short-lived recoil-fission events of transfer reaction products such as ${ }^{242 m} \mathrm{Am}$.

The thirty identified chains contain five recoil- $\alpha-\alpha$ fission and two recoil- $\alpha$-fission events, which are compatible with decay chains proposed to originate from either ${ }^{289} 115$ or ${ }^{288} 115$ [27,28]. One five- $\alpha$ long chain displayed as chain 23 on the right-hand side of Fig. 1 is clearly compatible with the characteristics of the decay chain attributed to start from the isotope ${ }^{287} 115$ [28,29]. None of these chains had Ge-detector events in prompt coincidence with $\alpha$ decays. The applied two-dimensional prompt coincidence window is shown on the upper left of Fig. 2.

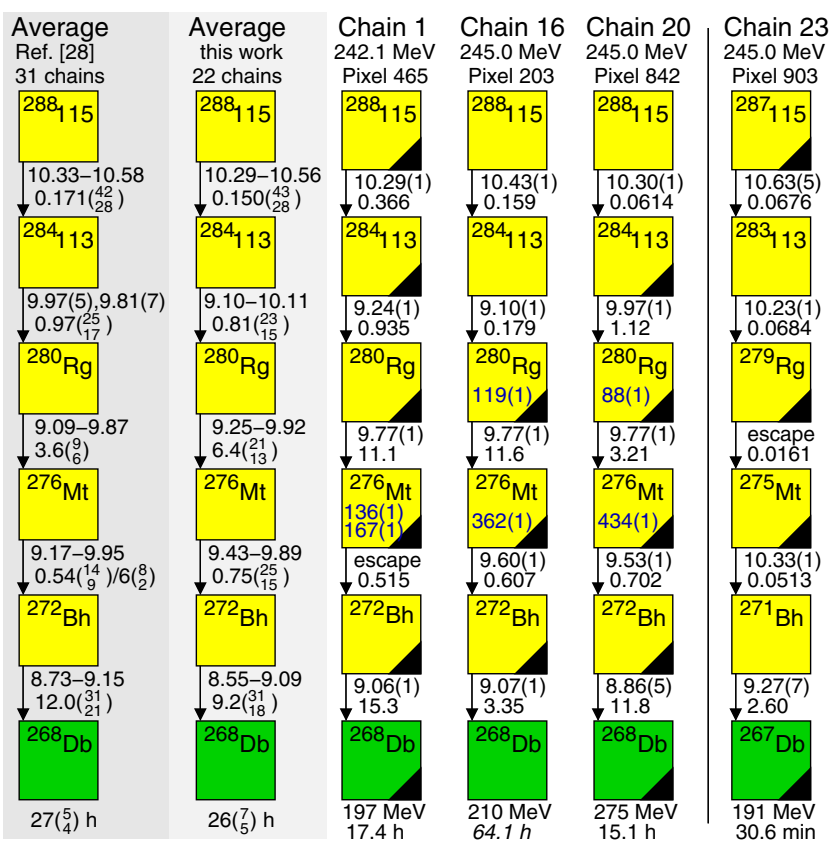

FIG. 1 (color online). Correlated decay chains of ${ }^{287,288} 115$ of the present work compared with Ref. [28]. Individual chains are labeled with an identification number, beam energy at midtarget, and a pixel identifier of the implantation detector. Decay energies are in $\mathrm{MeV}$ and times in seconds. Uncertainties of $0.01 \mathrm{MeV}$ for single measurements imply full-energy detection in the implantation DSSSD, otherwise the energy was reconstructed from coincidences in the implantation and one box DSSSD. Cases marked "escape" denote events with low-energy detection solely in the implantation DSSSD. Numbers with uncertainties inside the yellow squares relate to photon energies detected in prompt coincidence with the respective $\alpha$-decay mother. Black triangles indicate detection during beam-off periods. 

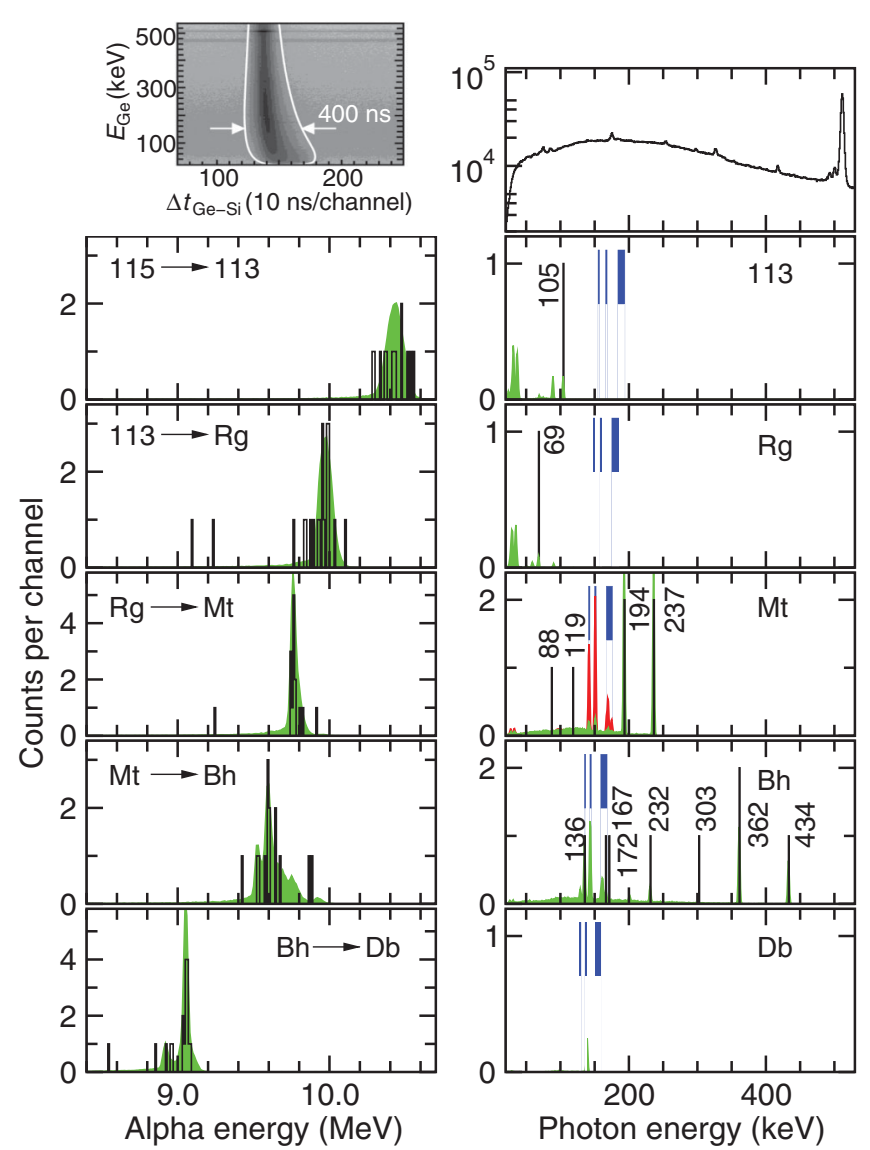

FIG. 2 (color). Top row: correlation time between events in the implantation DSSSD (left) and any of the Ge detectors as a function of Ge-detector energy, and the total projection of Ge-detector events in prompt coincidence with events in the implantation DSSSD (right), both accumulated during beam-off periods. The prompt coincidence window is indicated by the white line in the two-dimensional plot. The remaining five rows show energy spectra of the DSSSD Si cube, and Ge-detector spectra in prompt coincidence with the DSSSD events for each $\alpha$-decay step starting from ${ }^{288} 115$ (black histograms). The blue vertical bars in the photon spectra indicate predicted $K_{\alpha 2}, K_{\alpha 1}$, and $K_{\beta}$ energies of the corresponding element. Peak labels mark photon energies in $\mathrm{keV}$. The smooth curves (green and red) show scaled Monte-Carlo simulations of $10^{5} 288115$ ions implanted into a virtual detector setup [32] using decay schemes as those in Fig. 3.

The remaining 22 chains are fully or in significant portions compatible with the 31 chains previously assigned to the decay of ${ }^{288} 115$ [28,29]. Figure 1 compares averaged values reported from FLNR [28] with our values, shown together with three selected chains, which are considered most relevant for the construction of decay schemes and $\mathrm{x}$-ray fingerprinting. A table with detailed information on our results is available as Supplemental Material [30]. For a comprehensive assessment, see Ref. [27].

The left column of Fig. 2 provides five energy spectra taken with the DSSSD cube in the energy range of interest.
They comprise both full-energy and reconstructed events (cf. caption Fig. 1) of the respective step in the $\alpha$-decay chains, starting with ${ }^{288} 115$.

The topmost spectrum of the right column shows the total number of counts in all Ge-detector crystals during beam-off periods $\left(t_{\text {beam-off }}=1.10 \times 10^{6} \mathrm{~s}\right)$ taken in prompt coincidence with events at any energy detected above threshold in the implantation DSSSD. It has $N_{\gamma}=8.0 \times$ $10^{6}$ data points in the displayed energy range, dominated by the $511-\mathrm{keV} e^{+} e^{-}$annihilation peak. The remaining five spectra in the right column provide Ge-detector spectra taken in prompt coincidence with the corresponding, firmly established $\alpha$ decays along the chains. These spectra have $1,1,6,8$, and 0 counts, respectively. For a given pixel, e.g., No. 465 from chain 1 (cf. Fig. 1), and escapelike energies, $0.5<E_{\mathrm{Si}}<2.0 \mathrm{MeV}$, the corresponding prompt Ge-detector spectrum has 5620 events, i.e., a coincidence rate of about 1 in $200 \mathrm{~s}$ during beam-off periods. Selecting detected full-energy and reconstructed $\alpha$ particles instead, $8.0<E_{\mathrm{Si}}<12.0 \mathrm{MeV}$, these numbers reduce to four counts, hence 1 such coincidence per $75 \mathrm{~h}$. The expected low random rate is nicely corroborated by the absence of $511-\mathrm{keV}$ events in any of the five spectra of interest. Nevertheless, there is a finite probability of $10 \%-15 \%$ for random $\alpha$-photon coincidences [27], corresponding to about 2 of the 16 photon events in Fig. 2. Another random photon may arise from an unrelated escapelike event possibly being assigned as a true member to any of the present 22 chains (cf. the Supplemental Material [27,30]).

Only recently, realistic GEANT4 Monte Carlo simulations have been introduced to support conclusions drawn from experimental data on heavy nuclei [31]. To start with, the simulations provide proper implantation profiles of the ions of interest, followed by tracking recoil and decay energies. They account for transition probabilities of nuclear and atomic deexcitations, including emission and detection of $\alpha$ particles, $\gamma$ rays, conversion electrons, $\mathrm{x}$ rays, and Auger electrons. In particular, possible energy summing of interactions of different types of decay radiation within a given active detector element of the setup is handled properly.

Assuming production and decay of 288115 , the chains of $10^{5}$ ions implanted into a virtual TASISpec [32] were simulated. The decay schemes of the isotopes along the chain are the physics input (cf. Fig. 3). The output is organized such that it produces list mode data, i.e., energy and time of hits in a given TASISpec detector element. This data set was sent through the same offline analysis codes as used for the calibrated experimental data. The simulated energy spectra are displayed by smooth curves in the lower five rows of Fig. 2. One common normalization factor is used for all simulated energy spectra, such that the ratio between the number of counts in simulated and measured $\alpha$-decay spectra is on average 2. 


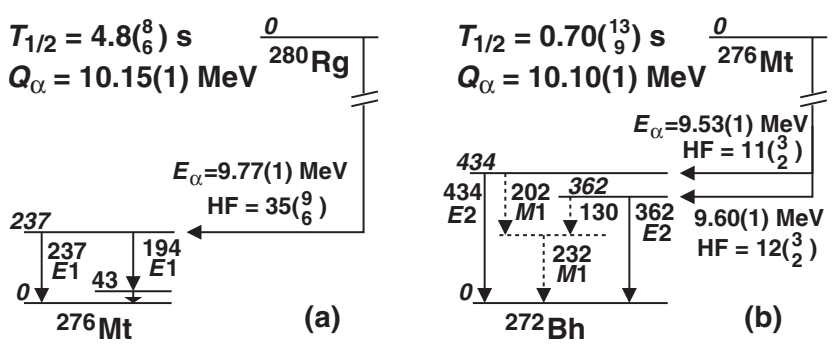

FIG. 3. Proposed decay schemes of ${ }^{280} \mathrm{Rg} \rightarrow{ }^{276} \mathrm{Mt}$ (a) and ${ }^{276} \mathrm{Mt} \rightarrow{ }^{272} \mathrm{Bh}(\mathrm{b})$. Level and $\gamma$-ray energies are in $\mathrm{keV}$, tentative levels and transitions are dashed. $E 1, M 1$, and $E 2$ denote proposed $\gamma$-ray multipolarities. $\alpha$-decay half-lives $T_{1 / 2}$ are based on present and previously published $[28,37]$ data. $\mathrm{HF}=T_{1 / 2}^{\exp } / T_{1 / 2}^{\text {sys }}$ are hindrance factors with $T_{1 / 2}^{\mathrm{sys}}$ calculated according to Ref. [38].

The broad distribution of $\alpha$-decay energies and the halflife of ${ }^{288} 115$ are compatible with Ref. [28]. To explain this distribution and the possible photon event, the simulation of the ${ }^{288} 115$ decay suffices with one $\alpha$-decay branch of $E_{\alpha}=10.30(1) \mathrm{MeV}$ to an excited state, which cascades down towards the ground state of ${ }^{284} 113$ via a few highly converted $\gamma$-ray transitions with energies below the $Z=$ $113 K$-binding energy. This implies summing of $\alpha$ decay and one or more conversion or Auger electron energies. The simulation suggests $Q_{\alpha} \approx 10.7 \mathrm{MeV}$. From all known detected chains $T_{1 / 2}=0.16\left(\begin{array}{l}3 \\ 2\end{array}\right) \mathrm{s}$ is derived. The decay pattern of ${ }^{284} 113$ is very similar, leading to $Q_{\alpha} \approx 10.3 \mathrm{MeV}$ and $T_{1 / 2}=0.92\left(\begin{array}{c}16 \\ 12\end{array}\right) \mathrm{s}$.

The $\alpha$-decay results of ${ }^{280} \mathrm{Rg}$ agree with Ref. [28]. The photon spectrum associated with ${ }^{276} \mathrm{Mt}$ contains two counts below the $K$-shell binding energy of Mt, and two each at 194 and $237 \mathrm{keV}$. All six are in coincidence with full-energy $\alpha$ events. This leads to the decay scheme proposed in Fig. 3(a): A single $E_{\alpha}=9.77(1)-\mathrm{MeV}$ line populates an excited state at $237 \mathrm{keV}$ in ${ }^{276} \mathrm{Mt}$, which emits $E 1$ transitions of 194 and $237 \mathrm{keV}$ with about the same probability. The absence of $K$-x rays in the Ge-detector spectrum calls for the $E 1$ assignment of the two transitions based on low total conversion coefficients of $\alpha_{\text {tot }} \approx 0.1$ [33], while the 194-keV decay is followed by a possibly delayed, but certainly highly converted $43-\mathrm{keV}$ transition. The simulations based on these premises are compatible with both energy spectra of this decay step (green curves). Properly normalized, they also suggest 2.9 Comptonscattered and $0.4 K$ x-ray events in the photon energy regime $20-180 \mathrm{keV}$. A simulation assuming $M 1$ multipolarity of the $\gamma$ rays fails to reproduce the observed photon spectrum (red curve).

The decay scheme of ${ }^{276} \mathrm{Mt}$ in Fig. 3(b) features two $\alpha$-decay lines followed by a more complex $\gamma$-ray decay scheme, including the opportunity for observing Bh $K$-x rays. Chains 16 and 20 displayed in Fig. 1 establish two branches and $Q_{\alpha}=10.10$ (1) MeV. A second count at $362(1) \mathrm{keV}$ in the photon spectrum of ${ }^{272} \mathrm{Bh}$ is coincident with an escape event, which is properly embedded between mother and daughter decays. Similarly, the escape event in chain 1 is also firmly attributed to the decay of ${ }^{276} \mathrm{Mt}$ (cf. Fig. 1). It is coincident with two Ge-detector entries at 136 and $167 \mathrm{keV}$, which are consistent with $Z=107$ $K_{\alpha 2}$ and $K_{\beta}$ energies, respectively [16,17]. It is noteworthy that no other $Z$ assignment would generate two coincident $K$-x rays at these energies. However, either one or both of them can also represent $\gamma$ rays or Compton scattered or background events.

Assuming the observation of two coincident photons implies $\gamma$-decay cascades parallel to the 362 and $434-\mathrm{keV}$ transitions. From chain 5, an $E_{\alpha}=9.65(1) \mathrm{MeV}, E_{\gamma}=$ 232(1)-keV coincidence suggests a tentative state at $232 \mathrm{keV}$, which may connect to the 362 and 434-keV levels via 130 and $202-\mathrm{keV}$ transitions. The latter gives rise to a possible cascade of two $K$-converted $M 1$ transitions. Besides missing counts at the $K_{\alpha 1}$ energy, the normalized spectra of the simulation based on the partially tentative ${ }^{272}$ Bh decay scheme in Fig. 3(b) are compatible with the corresponding energy spectra in Fig. 2. The two possible Compton events of multiplicity one at 172 and $303 \mathrm{keV}$ are in line with expected 2.4 Compton scattered events in the range $E_{\gamma}=[170,350] \mathrm{keV}$. Note that $303=136+167$ or $303 \approx 144+160$, i.e., the sum of two $\mathrm{Bh} K$-x rays. Though detected in the Ge detector closest to the pixel in which the chain was observed, the simulation shows no enhancement at that energy; i.e., most likely it indeed represents a Compton scattered photon.

The energy spectrum and half-life of ${ }^{272} \mathrm{Bh}$ match with Ref. [28]. A distinct peak is observed, together with a distribution of $\alpha$-decay energies towards lower energies. A simulation based on a $75 \% E_{\alpha}=9.07(1) \mathrm{MeV}$ ground state to ground-state decay and a $25 \% E_{\alpha}=8.93(1) \mathrm{MeV}$ decay into an excited state at $E_{x} \approx 140 \mathrm{keV}$, which decays by a $140 \mathrm{keV} M 1$ or $E 2$ transition, provides a nearly perfect description of the experimentally observed pattern. The simulated $\gamma$-ray yield is marginal and compatible with an empty Ge-detector spectrum. This interpretation yields $Q_{\alpha}=9.21(1) \mathrm{MeV}$. A half-life $T_{1 / 2}=10.9\left(\begin{array}{c}21 \\ 15\end{array}\right) \mathrm{s}$ is derived from all available data points.

Many nuclear structure models (e.g., Refs. [34-36]) predict a shape change from near sphericity $(Z=115$ and 113) towards pronounced prolate deformation of $\beta_{2} \approx$ $\epsilon_{2} \approx 0.2$ (Mt, Bh, Db). Directly inferred experimental $Q_{\alpha}$ values provide a profound testing ground for the models along the complete ${ }^{288} 115 \alpha$-decay chain [27]. Hindrance factors (cf. Fig. 3) point toward favored $\alpha$ decays along the chain. Interestingly, at the above mentioned deformation, there is only one pair each of proton and neutron Nilsson single-particle orbitals near the Fermi surface that could generate the $E 1$ transitions observed in ${ }^{276} \mathrm{Mt}$. For instance, an excited $\nu[716] 13 / 2^{-} \otimes \pi[521] 1 / 2^{-}, I^{\pi}=6^{+}$state can couple to the $\nu[606] 11 / 2^{+} \otimes \pi[521] 1 / 2^{-}, I^{\pi}=5^{-}, 6^{-}$ doublet. In such a scenario, the odd $\pi[521] 1 / 2$ proton 
continues to act as a spectator in the decay to ${ }^{272} \mathrm{Bh}$. Here, the up-sloping neutron orbitals $\nu[606] 11 / 2$ and $\nu[604] 9 / 2$ stemming from the $g_{9 / 2}$ and $i_{11 / 2}$ shells, are close to the $\nu[613] 5 / 2$ orbital linked to the $d_{5 / 2}-g_{7 / 2}$ shells. Transitions between these orbitals can explain the proposed $\gamma$-decay scheme of ${ }^{272} \mathrm{Bh}$.

In conclusion, thirty correlated $\alpha$-decay chains were observed following the reaction ${ }^{48} \mathrm{Ca}+{ }^{243} \mathrm{Am}$. Decay schemes arising from high-resolution spectroscopic coincidence data, in conjunction with comprehensive Monte Carlo simulations, open the door for direct nuclear structure insights of these heaviest man-made atomic nuclei. Previous assignments linking the majority of the decay chains to the decay of ${ }^{287,288} 115$ [28,29] are confirmed. This includes first candidates for $Z$ fingerprinting the decay of Mt by means of characteristic $K$-x ray detection. There is clearly potential for direct determination of the atomic number of the descendants of superheavy elements.

The authors would like to thank the ion-source and the accelerator staff at GSI. This work is supported by the European Community FP7-Capacities ENSAR No. 262010, the Royal Physiographic Society in Lund, the Swedish Research Council, the German BMBF, the Office of Nuclear Physics, U.S. Department of Energy, and the UK Science and Technology Facilities Council.

*Dirk.Rudolph@nuclear.lu.se

[1] Y. Oganessian, J. Phys. G 34, R165 (2007).

[2] Yu. Ts. Oganessian et al., Phys. Rev. Lett. 104, 142502 (2010).

[3] Yu. Ts. Oganessian, Radiochim. Acta 99, 429 (2011).

[4] S. Hofmann et al., Eur. Phys. J. A 32, 251 (2007).

[5] L. Stavsetra, K. E. Gregorich, J. Dvorak, P. A. Ellison, I. Dragojević, M. A. Garcia, and H. Nitsche, Phys. Rev. Lett. 103, 132502 (2009).

[6] R. Eichler et al., Radiochim. Acta 98, 133 (2010).

[7] P. A. Ellison et al., Phys. Rev. Lett. 105, 182701 (2010).

[8] Ch.E. Düllmann et al., Phys. Rev. Lett. 104, 252701 (2010).

[9] J. M. Gates et al., Phys. Rev. C 83, 054618 (2011).

[10] S. Hofmann et al., Eur. Phys. J. A 48, 62 (2012).

[11] R. C. Barber, P. J. Karol, H. Nakahara, E. Vardaci, and E. W. Vogt, Pure Appl. Chem. 83, 1485 (2011).

[12] G. T. Seaborg and W. D. Loveland, The Elements Beyond Uranium (Wiley-Interscience, New York, 1990).

[13] R.-D. Herzberg and P.T. Greenlees, Prog. Part. Nucl. Phys. 61, 674 (2008).

[14] H. G. J. Moseley, Philos. Mag. 26, 1024 (1913).
[15] T.A. Carlson, C. W. Nestor, Jr., F.B. Malik, and T.C. Tucker, Nucl. Phys. A135, 57 (1969).

[16] B. Fricke and G. Soff, At. Data Nucl. Data Tables 19, 83 (1977).

[17] T. A. Carlson and C. W. Nestor, Jr., At. Data Nucl. Data Tables 19, 153 (1977).

[18] N. Gaston, P. Schwerdtfeger, and W. Nazarewicz, Phys. Rev. A 66, 062505 (2002).

[19] F. P. Heßberger et al., Eur. Phys. J. A 41, 145 (2009).

[20] C. E. Bemis, Jr., R. J. Silva, D. C. Hensley, O. L. Keller, Jr., J. R. Tarrant, L. D. Hunt, P.F. Dittner, R. L. Hahn, and C. D. Goodman, Phys. Rev. Lett. 31, 647 (1973).

[21] M. Schädel, Eur. Phys. J. D 45, 67 (2007).

[22] A. Semchenkov et al., Nucl. Instrum. Methods Phys. Res., Sect. B 266, 4153 (2008).

[23] J. Khuyagbaatar et al., Nucl. Instrum. Methods Phys. Res., Sect. A 689, 40 (2012).

[24] L.-L. Andersson et al., Nucl. Instrum. Methods Phys. Res., Sect. A 622, 164 (2010).

[25] U. Forsberg et al., Acta Phys. Pol. 43, 305 (2012).

[26] K. E. Gregorich, Nucl. Instrum. Methods Phys. Res., Sect. A 711, 47 (2013).

[27] U. Forsberg et al. (to be published).

[28] Yu. Ts. Oganessian et al., Phys. Rev. C 87, 014302 (2013).

[29] Yu. Ts. Oganessian et al., Phys. Rev. C 69, 021601(R) (2004).

[30] See Supplemental Material at http://link.aps.org/ supplemental/10.1103/PhysRevLett.111.112502 for a table which provides more detailed information on the decay chains presented and discussed in the present Letter.

[31] A. Lopez-Martens et al., Nucl. Phys. A852, 15 (2011).

[32] L. G. Sarmiento, L.-L. Andersson, and D. Rudolph, Nucl. Instrum. Methods Phys. Res., Sect. A 667, 26 (2012).

[33] T. Kibédi, T. W. Burrows, M. B. Trzhaskovskaya, P. M. Davidson, and C. W. Nestor, Jr., Nucl. Instrum. Methods Phys. Res., Sect. A 589, 202 (2008).

[34] P. Möller, J.R. Nix, W. D. Myers, and W. J. Swiatecki, At. Data Nucl. Data Tables 59, 185 (1995).

[35] S. Ćwiok, W. Nazarewicz, and P. H. Heenen, Phys. Rev. Lett. 83, 1108 (1999).

[36] S. Ćwiok, P. H. Heenen, and W. Nazarewicz, Nature (London) 433, 705 (2005).

[37] The long-lived $\alpha$ decays assigned to ${ }^{276} \mathrm{Mt}$ in chains 10 and 23 in Table II in Ref. [28] can be unanimously transferred to ${ }^{272} \mathrm{Bh}$ and ${ }^{280} \mathrm{Rg}$ as well, which puts the proposed $6 s$ decay branch of ${ }^{276} \mathrm{Mt}$ into doubt. Our combined correlation-time analysis of only one half-life excludes these two numbers.

[38] C. Qi, F. R. Xu, R. J. Liotta, R. Wyss, M. Y. Zhang, C. Asawatangtrakuldee, and D. Hu, Phys. Rev. C80, 044326 (2009). 